COMMENT. Summarizing the phenotypic profile of infants with STXBP1 mutations: an early-onset epilepsy within the first 5 months of life, beginning as Ohtahara syndrome or as EOEE, frequent evolution to West syndrome, severe mental retardation, and neurologic deficits with dyskinesia.

Mutations in STXBP1 occur in patients with Ohtahara and West syndromes and are also present in $10 \%$ of patients with undefined early-onset epileptic encephalopathies without the burst-suppression EEG, typical of Ohtahara syndrome. The authors recommend STXBP1 mutation analysis in the evaluation of infants with unexplained EOEE. Other known genes for epileptic encephalopathies include SCN1A, ARX, CDKL5, and PCDH19. Neurodegeneration and mental retardation associated with STXBP1 are presumed to be an intrinsic property of the gene mutations, and are independent of the severity of the epilepsy and response to antiepileptic drugs.

Genetic causes of epileptic syndromes will be added to the clinical and EEG classifications of the epilepsies as a result of expanding molecular research. Holland $\mathrm{KD}$ and Hallinan BE of Cincinnati, in an editorial (Neurology 2010;75:1132-1133), recommend genetic testing for infants with unexplained epileptic encephalopathies. A gene mutation diagnosis may obviate the necessity for exhaustive and invasive investigations and lead to more effective therapy. Dravet syndrome, an encephalopathy caused by mutations in the SCN1A sodium channel gene, is exacerbated by sodium channel AEDs (eg carbamazepine) (reviewed by Millichap JJ et al. Neurology 2009;73:e59-62). Seizures caused by STXBP1 mutations respond to vigabatrin and are resistant to other AEDs. (Deprez L et al. 2010).

\title{
EARLY-ONSET EPILEPTIC ENCEPHALOPATHY WITH PHOSPHOLIPASE C BETA 1 DEFICIENCY
}

The clinical presentation and evolution of epileptic encephalopathy associated with a loss-of-function mutation in the phospholipase C-b 1 gene are reported in a male infant with infantile spasms treated at the University of Birmingham School of Medicine, UK. The infant's parents were consanguineous. He presented with eye rolling, lip smacking, tonic stiffening and flexion seizures at 10 weeks, and subsequently developed infantile spasms and hypsarrhythmia at 8 months of age, associated with severe neurological regression (West syndrome). Spasms were refractory to a 2-week course of vigabatrin but were controlled with a course of prednisolone (ACTH was declined). At 10 months he developed tonic-clonic seizures resistant to AEDs and at 13 months, the EEG showed generalized slowing, consistent with diffuse encephalopathy. MRIs at ages 5 and 13 months were normal. His head circumference was at the $0.4^{\text {th }}$ centile at birth and at 2.5 years, when he was functioning at $0-3$ month level and was unable to lift his head when prone or roll over. At 2.9 years he had spastic quadriparesis, he contracted adenovirus pneumonitis and died of respiratory failure. Post-mortem was not performed. Molecular genetic analysis of the index case revealed a homozygous deletion on chromosome 20 involving the phospholipase PLCB1 gene. Linkage to the phospholipase C-b 1 locus was absent in 12 other consanguineous families of children with infantile spasms. The findings were consistent with genetic heterogeneity in infantile spasms. This is the first loss of function PLCB1 mutation described in humans. (Kurian MA, Meyer E, Vassallo $\mathrm{G}$, et al. Phospholipase $\mathrm{C}$ beta 1 deficiency is associated with early-onset epileptic 
encephalopathy. Brain Oct 2010;133(10):2964-2970). (Respond: Eamonn R Meyer, Department of Medical and Molecular Genetics, University of Birmingham School of Medicine, Institute of Biomedical Research, Birmingham, B15 2TT, UK. E-mail: e.r.meyer@bham.ac.uk).

COMMENT. Infantile spasms and West syndrome is a heterogeneous disorder with cryptogenic and symptomatic etiologies. Genetic causes for West syndrome listed by the authors include mutations of ARX, controlling development of g-aminobutyric acid-ergic interneurons, mutations in STXBP1 (in Ohtahara and West syndromes), and deletions involving MAG12. Phospholipase B-b 1 gene is involved in hippocampal muscarinic acetylcholine receptor signaling and in cortical development. The discovery of this mutation uncovers a novel mechanism for early-onset epileptic encephalopathy, infantile spasms and West syndrome.

\section{EEG AND MR SPECTROSCOPY IN HYPOXIC-ISCHEMIC ENCEPHALOPATHY IN TERM NEWBORNS}

Researchers from the University of Bologna, Italy, studied the relation of amplitude integrated EEG findings in the first $24 \mathrm{hrs}$ of life to brain metabolic changes, detected by proton MR spectroscopy (H-MRS) at 7-10 days of life, in 32 term newborns with hypoxic-ischemic encephalopathy (HIE). a-EEG at 6, 12 and $24 \mathrm{hrs}$ of life was significantly correlated with outcome, and showed improvement in newborns with normal H-MRS and good outcome and a deterioration in those with abnormal H-MRS and poor outcome. a-EEG time course documents the severity, as defined by H-MRS, and evolution of brain damage following an HI event in non-cooled newborns. Both a-EEG and MRS are correlated with outcome.

At follow-up till 2 years of age in 31 surviving patients, $21(67.7 \%)$ had a normal motor outcome, 7 (22.6\%) developed transitory hypertonia, and $3(9.7 \%)$ developed CP. Newborns with normal motor outcome or transitory hypertonia had a mean General Quotient of 104 on the Griffiths Mental Development Scale. BAER was normal in all cases; a cortical visual impairment occurred in 2 of 3 patients with $\mathrm{CP}$. Eight patients $(25 \%)$ developed neonatal seizures; the a-EEG background was severely abnormal in 3 and moderately abnormal in 5 cases at 6 hrs of life. Newborns with a normal a-EEG background at $6 \mathrm{hrs}$ developed normally, whereas 2 with severely abnormal EEG background had a poor prognosis ( 1 died, 1 had $\mathrm{CP}$ ). Of the 5 with moderately abnormal EEG background pattern at $6 \mathrm{hrs}, 2$ with a deteriorating pattern at $12 \mathrm{hrs}$ developed CP, whereas 3 unchanged at $12 \mathrm{hrs}$ and recovered at $24 \mathrm{hrs}$ showed a normal outcome. Electrographic seizures did not change prognostic accuracy of a-EEG background pattern. MRI abnormalities were observed in 5/31 patients. (Ancora G, Soffritti S, Lodi $\mathrm{R}$, et al. A combined a-EEG and MR spectroscopy study in term newborns with hypoxicischemic encephalopathy. Brain Dev November 2010;32:835-842). (Respond: Dr Gina Ancora, Neonatology Unit, Dept of Woman, Child and Adolescent Health, University of Bologna, Italy. E-mail: gina.ancora@unibo.it).

COMMENT. Seizure activity dedected at a-EEG in HIE newborns was associated with a poor outcome only in patients with abnormal a-EEG background pattern. Patients 